\title{
ON MIXING MEASURES FOR AXIOM A DIFFEOMORPHISMS
}

\author{
KARL SIGMUND
}

\begin{abstract}
Let $f$ be a diffeomorphism satisfying Smale's Axiom A and $X$ an infinite basic set such that $f \mid X$ is topologically mixing. Let $\mathscr{M}$ denote the space of $f$-invariant measures on $X$ with the weak topology. It is shown that for a dense set of measures $\mu$ in $\mathscr{M}$, the system $(X, f, \mu)$ is Bernoulli. It follows that "in general" the elements of $\mathscr{M}$ are weakly mixing.
\end{abstract}

1. Introduction. In [4], Bowen constructs an invariant measure for diffeomorphisms satisfying Smale's Axiom A which is in some sense the "best" measure, and in [5] he shows that under certain natural conditions the resulting dynamical system is a $K$-automorphism and, in fact, even measure theoretically isomorphic to a Bernoulli shift. In particular, it is strongly mixing and has positive entropy. In this note one considers the space of invariant measures provided with the weak topology and asks whether it contains many other measures with metric properties similar to those of Bowen's measure.

Bowen's theory of Markov partitions and results obtained in [14] allow one to approximate every invariant measure by Markov chains of positive entropy. If the diffeomorphism satisfies some mild condition, the approximating measures are strongly mixing and the corresponding dynamical system is measure theoretically isomorphic to a Bernoulli shift. As a corollary, one obtains that the invariant measures are generically weakly mixing.

In [7] and [16], theorems concerning invariant measures for Axiom A diffeomorphisms have been extended to the case of hyperbolic flows. One might expect that the results of this paper remain true for the space of invariant measures for $C$-dense Axiom A flows (see [7]).

2. Definitions and results. Let $M$ be a compact $C^{\infty}$ manifold without boundary and $f: M \rightarrow M$ a diffeomorphism. The nonwandering set $\Omega$ is the (closed, invariant) set of all $x \in M$ such that for every neighborhood $U$ of $x$, there is an $n \neq 0$ with $T^{n} U \cap U \neq \varnothing . f$ satisfies Axiom A if $\Omega$ is hyperbolic and the periodic points of $f$ are dense in $\Omega$ (see [17, p. 777]). One

Received by the editors November 29, 1971 and, in revised form, March 29, 1972. AMS 1970 subject classifications. Primary 28A65, 58F15.

Key words and phrases. Bernoulli shift, entropy, mixing measures, Markov partitions, Markov chains, quasiregular points.

(C) American Mathematical Society 1973 
can then write $\Omega$ as the finite disjoint union of basic sets $\Omega_{s}$, where each $\Omega_{s}$ is closed invariant and $f \mid \Omega_{s}$ is topologically transitive. By Bowen's $C$-density decomposition theorem [4, Theorem 2.7] one can further decompose each $\Omega_{s}$ into a finite disjoint union of closed sets $\Omega_{s, j}$ $\left(1 \leqq j \leqq m_{s}\right)$ with $f\left(\Omega_{s, j}\right)=\Omega_{s, j+1}\left(f\left(\Omega_{s, m_{s}}\right)=\Omega_{s, 1}\right)$ and $f^{m_{s} \mid} \Omega_{s, 1}$ topologically mixing. (Recall that a homeomorphism $g: X \rightarrow X$ is topologically mixing if for any pair of open sets $A, B \subset X$ there is an $N$ such that $g^{n}(A) \cap B \neq \varnothing$ for all $n \geqq N$.)

Every $f$-invariant (Borel) probability measure in $M$ has support in $\Omega$ and can be written as a finite convex combination of invariant measures concentrated on basic sets. Let $\Omega_{s}$ be a fixed basic set with infinitely many elements and let $\mathscr{M}$ denote the space of $f$-invariant probability measures concentrated in $\Omega_{s} . \mathscr{M}$ is provided with the weak topology for measures (see $[12$, p. 40]).

Bowen constructs in [4] an element of $\mathscr{M}$ which is nonatomic, positive on open sets, ergodic, and has positive entropy. (It is the unique measure maximizing entropy.) In [14] it was shown that there is a dense $G_{\delta}$ subset $\mathscr{N} \subset \mathscr{M}$ such that if $\mu \in \mathscr{N}^{\prime}$, then $\mu$ is nonatomic, positive on open sets, ergodic, and has entropy zero.

No $\mu \in \mathscr{M}$ can be weakly mixing if $f \mid \Omega_{s}$ is not topologically mixing. But the restriction to the topologically mixing case is very natural: to

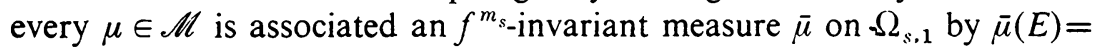
$m_{s} \cdot \mu(E)$ for all Borel sets $E \subset Q_{s .1}$, and the correspondence $\mu \rightarrow \bar{\mu}$ is a homeomorphism. The system $\left(\Omega_{s}, f, \mu_{13}\right)$, where $\mu_{B}$ denotes Bowen measure, is a $K$-automorphism iff $f \mid \Omega_{s}$ is topologically mixing (see [5, Theorem 34]). In this case, $\left(\Omega_{s}, f, \mu_{B j}\right)$ is measure theoretically isomorphic to a strongly mixing Markov chain and hence, by [9], to a Bernoulli shift. In [14] it was shown that strongly mixing measures form a set of first category in $\mathscr{M}$. The following theorem shows that they are dense.

THEOREM. If $f \mid \Omega_{s}$ is topologically mixing, then the measures $\mu$ such that $\left(\Omega_{s}, f, \mu\right)$ is measure theoretically isomorphic to a Bernoulli shift form a dense subset of $\mathscr{M}$.

COROLlaRY 1. If $f \mid \Omega_{s}$ is topologically mixing then the measures which are mixing of order $n$ are dense in $\mathscr{M}($ for all $n)$. The set of weakly mixing measures is a dense $G_{\delta}$ in $\mathscr{M}$.

COROllary 2. The set of measures with positive entropy is dense in $\mathscr{M}$.

Hyperbolic automorphisms of the $n$-torus are examples of Axiom A diffeomorphisms with only one basic set $\Omega_{s}$ and $f \mid \Omega_{s}$ topologically mixing.

In [5], Bowen defines a continuous equivariant map $\pi$ from a zerodimensional dynamical system $\Sigma$ onto $\Omega_{s}$. The properties of $\pi$ play a 
central role in the proof of the theorem above. Bowen shows in [6] that $x \in \Sigma$ is periodic (or recurrent, or topologically transitive) iff $\pi(x)$ is periodic (or recurrent, or topologically transitive). One can look for further properties preserved under $\pi$. At the end of this paper there is a construction of a quasiregular $q \in \Omega_{s}$ such that $\pi^{-1}(q)$ contains a point which is not quasiregular.

3. Proofs. Let $S$ denote the discrete space $\{1, \cdots, S\}$ and $O(S)$ the space of all functions $x: i \leadsto x_{i}$ from the integers $Z$ into $S$, provided with the product topology. Let $\sigma$ denote the shift homeomorphism of $\theta(S)$ :

$$
[\sigma(x)]_{i}=x_{i+1} \text { for } x \in \theta(S) .
$$

Let $T=\left(t_{i j}\right)$ be an $S \times S$-matrix of zeroes and ones. An element $x$ of $\theta(S)$ is said to be $T$-admissible if $t_{x_{i} \cdot x_{i \cdot 1}}=1$ for all $i \in Z$. One similarly defines $T$-admissible blocks $x_{1} x_{2} \cdots x_{k}$ of elements of $S$. The set $\Sigma$ of all $T$-admissible $x \in \theta(S)$ is a compact metrizable space invariant under $\sigma .(\Sigma, \sigma)$ is called the subshift of finite type associated with $T$.

In [5] Bowen proves that to every basic set $\Omega_{s}$ of the Axiom A diffeomorphism $f$ there corresponds a subshift of finite type $(\Sigma, \sigma)$ and a continuous surjective map $\pi: \Sigma \rightarrow \Omega_{s}$ such that the diagram

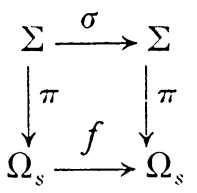

commutes.

Lemma 1 [6, Corollary 11]. If $x \in \Omega_{*}$ is periodic, then every $y \in \pi^{-1}(x)$ is periodic.

Lemma 2 [5, Proposition 30]. If $f \mid \Omega_{s}$ is topologically mixing, then $(\Sigma, \sigma)$ is also topologically mixing.

It is easy to see that $(\Sigma, \sigma)$ is topologically mixing iff $T$ is irreducible and aperiodic (i.e. iff there is an $N$ such that $t_{i j}^{(n)}>0$ for all $n>N$ and all $i, j)$.

If $g$ is a homeomorphism of some space $X$ and $x \in X$ a periodic point of period $k$, then the $g$-invariant measure $\mu_{x}$ which has mass $1 / k$ at the points $x, g x, \cdots, g^{k-1} x$ is called a periodic orbit measure (p.o.-measure).

Lemma 3. If $(\Sigma, \sigma)$ is a subshift of finite type with periodic points dense and $\mu$ is an invariant measure, then $\mu$ can be approximated by p.o.-measures $\mu_{x}$. If $\Sigma$ has infinitely many elements, the period of $x$ can be chosen arbitrarily large and prime. 
Proof. By [3, Theorem 6.7], $(\Sigma, \sigma)$ is topologically conjugate to a basic set of some Axiom A diffeomorphism of the 2-sphere. The lemma follows by [14, Theorem 1 and Lemma 1].

LEMMA 4. If $(\Sigma, \sigma)$ is a topologically mixing subshift of finite type with periodic points dense and $V(\mu)$ is a neighborhood of the invariant measure $\mu$ on $\Sigma$, then $V(\mu)$ contains a $\rho$ such that the system $(\Sigma, \sigma, \rho)$ is measure theoretically isomorphic to a Bernoulli shift.

Proof. By Lemma 3, there is a periodic point $x \in \Sigma$ of prime minimal period $p$ such that $\mu_{x} \in V(\mu)$. Let $S^{\prime}$ denote the set of all $T$-admissible $p$-blocks of elements of $S$, arranged in such a way that the first $p$ of the $S^{\prime}$ elements of $S^{\prime}$ are $\left(x_{1} \cdots x_{p}\right),\left(x_{2} \cdots x_{p} x_{1}\right), \cdots,\left(x_{p} x_{1} \cdots x_{p-1}\right)$ (in this order). The transition rules given by $T$ specify transition rules between the states of $S^{\prime}$. Let $T^{\prime}=\left(t_{i j}^{\prime}\right)$ denote the resulting $S^{\prime} \times S^{\prime}$ transition matrix and $\Sigma^{\prime}$ the corresponding subshift of $\theta\left(S^{\prime}\right)$. Since $\left(\Sigma^{\prime}, \sigma\right)$ is topologically conjugate to $(\Sigma, \sigma)$, the matrix $T^{\prime}$ is irreducible and aperiodic. Let us write

$$
T^{\prime}=\left[\begin{array}{ll}
A_{0} & B_{0} \\
C_{0} & D_{0}
\end{array}\right]
$$

where $A_{0}$ is a $p \times p$-submatrix. Since $p$ is prime, $A_{0}$ must be of the form

$$
\left[\begin{array}{ccccc}
0 & 1 & 0 & \cdots & 0 \\
0 & 0 & 1 & \cdots & 0 \\
\cdot & & & & \cdot \\
\cdot & & & & \cdot \\
\cdot & & & & \cdot \\
0 & 0 & 0 & \cdots & 1 \\
1 & 0 & 0 & \cdots & 0
\end{array}\right] .
$$

Indeed, if $\left(x_{j} x_{j+1} \cdots x_{j-1}\right)$ is a successor of $\left(x_{i} x_{i+1} \cdots x_{i-1}\right)$ according to the transition rules given by $T^{\prime}$, it follows that $x_{i+1}=x_{j}$, and generally $x_{m}=x_{n}$ if $m-n=i+1-j(m, n$ modulo $p)$. If $j \neq i+1$, one would obtain $x_{1}=x_{2}=\cdots=x_{p}$, a contradiction to the choice of $x$. Since $\Sigma$ is infinite, one has furthermore that $A_{0}$ is a proper submatrix of $T^{\prime}$. Therefore, there exists a stochastic $S^{\prime} \times S^{\prime}$ matrix $L=\left(l_{i j}\right)$ of the form

$$
L=\left[\begin{array}{ll}
A_{0} & 0 \\
C_{1} & D_{1}
\end{array}\right]
$$


such that, for all $(i, j)$ with $i>p$ or $j \leqq p$, one has $l_{i j}>0$ iff $t_{i j}^{\prime}>0$. For $n>0$ one has

$$
L^{n}=\left[\begin{array}{cc}
A_{0}^{n} & 0 \\
E(n) & D_{1}^{n}
\end{array}\right] .
$$

The elements of $E(n)$ depend only on the $l_{i j}$ with $i>p$ or $j \leqq p$. Since these $l_{i j}$ vanish iff the corresponding $t_{i j}^{\prime}$ vanish, and since $T^{\prime}$ is irreducible and aperiodic, there is an $N$ such that all elements of $E(n)$ are positive for $n \geqq N$.

The stochastic matrix $L$ has at least one eigenvector $\lambda=\left(\lambda_{1}, \cdots, \lambda_{S^{\prime}}\right)$ for the eigenvalue 1 . Since $\lambda$ is also an eigenvector of $L^{n}$, the vector $\left(\lambda_{p+1}, \cdots, \lambda_{S^{\prime}}\right)$ is an eigenvector of $D_{1}^{n}$ for the eigenvalue 1 . But for $n \geqq N$, the row sums of $D_{1}^{n}$ (and hence its $L^{\infty}$-norm) are less than 1 , and therefore $\lambda_{j}=0$ for $p<j \leqq S^{\prime}$. Furthermore, the form of $A_{0}$ implies that $\lambda_{1}=$ $\lambda_{2}=\cdots=\lambda_{n}$. Hence the matrix $L$, although not irreducible, has only one normed eigenvector for the eigenvalue 1 , given by $\bar{\lambda}=(1 / p, \cdots, 1 / p$, $0, \cdots, 0)$. Remark that the invariant measure for $\left(\Sigma^{\prime}, \sigma\right)$ given by the Markov shift induced by the transition matrix $L$ and the initial probabilities $\bar{\lambda}$ (see [2, p. 30]) is just the measure $\mu_{x}$.

Consider now the stochastic $S^{\prime} \times S^{\prime}$-matrix $P$ of the form

$$
P=\left[\begin{array}{ll}
A_{1} & B_{1} \\
C_{1} & D_{1}
\end{array}\right]
$$

where $A_{1}$ is a $p \times p$-matrix given by

$$
\left[\begin{array}{ccccc}
0 & 1-\varepsilon_{1} & 0 & \cdots & 0 \\
0 & 0 & 1-\varepsilon_{2} & \cdots & 0 \\
\cdot & & & & \cdot \\
\cdot & & & & \cdot \\
\cdot & & & & \cdot \\
1-\varepsilon_{p} & 0 & 0 & \cdots & 0
\end{array}\right]
$$

and the elements of $B_{1}$ are positive iff the corresponding elements of the submatrix $B_{0}$ of $T^{\prime}$ are positive. Like $T^{\prime}, P$ is irreducible; hence, there exists a unique eigenvector $\bar{\pi}=\left(\pi_{1}, \cdots, \pi_{S}\right)$ for the eigenvalue 1 (see, for example, Feller [8, p. 393]). If one chooses $\varepsilon_{1}, \cdots, \varepsilon_{p}$ small enough, $P$ approximates $L$ and $\bar{\pi}$ approximates the unique normed eigenvector $\overline{\hat{\lambda}}$ corresponding to the eigenvalue 1 of $L$. Thus the invariant measure $\rho$ for $\left(\Sigma^{\prime}, \sigma\right)$ given by the Markov shift induced by $P$ and $\bar{\pi}$ can be chosen arbitrarily near the measure $\mu_{x}$ and therefore within $V(\mu)$. Since $P$ is 
irreducible and aperiodic, the system $\left(\Sigma^{\prime}, \sigma, \rho\right)$ is a mixing Markov shift and hence (see [9]) measure theoretically isomorphic to a Bernoulli shift. Hence Lemma 4 is proved.

Proof of The Theorem. Let $\mu \in \mathscr{M}$ and a neighborhood $V(\mu)$ of $\mu$ be given. By [14, Theorem 1] there is a p.o.-measure $\mu_{x}$ in $V(\mu)$. Let $y$ be an element of $\pi^{-1}(x)$. By Lemma $1, y$ is periodic. By Lemma 4 , there is a sequence of measures $\rho_{n}$ converging to the p.o.-measure $\mu_{y}$ such that all systems $\left(\Sigma, \sigma, \rho_{n}\right)$ are (isomorphic to) Bernoulli shifts. The measures $\pi\left(\rho_{n}\right)$ converge to $\pi\left(\mu_{y}\right)=\mu_{x}$ and the systems $\left(\Omega_{s}, f, \pi\left(\rho_{n}\right)\right)$ are-as homomorphic images of Bernoulli shifts-also Bernoulli shifts (see [10]). Hence $V(\mu)$ contains a $\rho \in \mathscr{M}$ such that $\left(\Omega_{s}, f, \rho\right)$ is measure theoretically isomorphic to a Bernoulli shift.

PrOOF OF COROLlaRY 1. The first part follows immediately from $[13$, p. 44]. The second follows from the proof of Theorem 2 in [11], which shows that if $\varphi$ is a homeomorphism of a compact metric space $X$, the set of weakly mixing measures is a $G_{\delta}$ in the set of $\varphi$-invariant measures on $X$.

Proof of Corollary 2. If $\left(\Omega_{s}, f, \rho\right)$ is a Bernoulli shift, the entropy $h_{\rho}(f)$ of $f$ with respect to $\rho$ is positive. If $f \mid \Omega_{s}$ is not topologically mixing, the corollary follows from Bowen's $C$-density decomposition theorem and the fact that $h_{\mu}\left(f^{k}\right)=k \cdot h_{\mu}(f)$ for $k>0$ and $\mu f$-invariant.

4. On quasiregular points. Let $X$ be a compact metric space and $p$ a homeomorphism $X \rightarrow X$. A point $q \in X$ is called quasiregular if

$$
\lim _{N \rightarrow \infty} \frac{1}{N} \sum_{i=0}^{N-1} g\left(\varphi^{i} q\right)
$$

exists for all continuous real-valued $g$ on $X$. The map

$$
g \rightarrow \lim _{N \rightarrow \infty} \frac{1}{N} \sum_{i=0}^{N-1} g\left(\varphi^{i} q\right)
$$

is a $\varphi$-invariant measure on $X$. In [15] it is shown that every $\mu \in \mathscr{M}$ is generated in this way by some quasiregular $q \in \Omega_{s}$.

One sees easily that if $q \in \Sigma$ is quasiregular, then $\pi(q)$ is quasiregular. We construct a $q \in \Sigma$ which is not quasiregular, but such that $\pi(q)$ is quasiregular.

Let $\left(\Omega_{s}, f\right)$-and hence also $(\Sigma, \sigma)$-be topologically mixing. Let $x$ denote a periodic point in $\Omega_{s}$ such that $\pi^{-1}(x)$ contains two periodic points $x^{(1)}$ and $x^{(2)}$ belonging to two different orbits.

(Such a situation occurs, for example, in [1, p. 30ff.], where $\left(\Omega_{s}, f\right)$ is the hyperbolic toral automorphism given by the matrix $A=\left[\begin{array}{cc}3 & -1 \\ 1\end{array}\right]$. In [1] it is shown that the associated subshift of finite type is given by 
the transition matrix

$$
\left[\begin{array}{lllll}
1 & 0 & 1 & 0 & 0 \\
1 & 0 & 1 & 0 & 0 \\
0 & 1 & 0 & 1 & 1 \\
0 & 1 & 0 & 1 & 1 \\
0 & 1 & 0 & 1 & 1
\end{array}\right] .
$$

Let $x$ be the fixed point of the torus, $x^{(1)}$ the sequence $(\cdots, 1,1,1, \cdots)$ and $x^{(2)}$ the sequence $(\cdots, 5,5,5, \cdots)$.)

One can assume that $x^{(1)}$ and $x^{(2)}$ have the same (not necessarily minimal) period $p$. Let $A^{(j)}$ denote the $T$-admissible block $x_{1}^{(j)} \cdots x_{p}^{(j)}$ $(j=1,2)$. Let $B_{12}$ denote a block of elements of $S$ such that the block $A^{(1)} B_{12} A^{(2)}$ is $T$-admissible. (Such a $B_{12}$ exists since $(\Sigma, \sigma)$ is topologically mixing.) Similarly, let $B_{21}$ be a block of elements of $S$ such that $A^{(2)} B_{21} A^{(1)}$ is $T$-admissible. Let $m=\max \left(\operatorname{card} B_{12}, \operatorname{card} B_{21}\right)$.

Let $q \in \theta(\boldsymbol{S})$ be given by

$$
\begin{array}{cc}
\cdots A^{(1)} A^{(1)} B_{12} \cdot A^{(2)} A^{(2)} \cdots A^{(2)} B_{21} & A^{(1)} A^{(1)} \cdots A^{(1)} \\
\left(k_{1} \text { copies }\right) & \left(k_{2} \text { copies }\right) \\
\times B_{12} A^{(2)} A^{(2)} \cdots A^{(2)} B_{21} \cdots \\
\left(k_{3} \text { copies }\right)
\end{array}
$$

(the dot denotes the decimal point between $q_{0}$ and $q_{1}$ ). Let $k_{j}$ be an increasing sequence of integers such that

$$
\frac{\left(k_{1}+k_{2}+\cdots+k_{j}\right) p+m j}{\left(k_{1}+k_{2}+\cdots+k_{j+1}\right) p}<2^{-j},
$$

$q$ is $T$-admissible, and hence in $\Sigma$. The iterates of $q$ under $\sigma$ follow the orbit of $x^{(2)}$ for a time, then the orbit of $x^{(1)}$ for a much longer time, then the orbit of $x^{(2)}$ for a still much longer time, etc. One can easily check that $q$ is not quasiregular. But since $\pi$ is continuous, and maps both $x^{(1)}$ and $x^{(2)}$ into $x$, the distance between $f^{n}(x)$ and $f^{n}(\pi(q))$ converges to 0 , if one restricts $n$ to the complement, in $Z^{+}$, of some set of density zero. It follows that $\pi(q)$ is quasiregular and generates the p.o.-measure $\mu_{x}$.

ACKNOWLEDGEMENT. The idea of approximating p.o.-measures by Markov chains is due to Parthasarathy [11]. The author wishes to thank Benjamin Weiss for helpful conversations. 


\section{REFERENCES}

1. R. L. Adler and B. Weiss, Similarity of automorphisms of the torus, Mem. Amer. Math. Soc. No. 98 (1970). MR 41 \#1966.

2. P. Billingsley, Ergodic theory and information, Wiley, New York, 1965. MR 33 \#254.

3. R. Bowen, Topological entropy and Axiom A, Proc. Sympos. Pure Math., vol. 14, Amer. Math. Soc., Providence, R.I., 1970, pp. 23-41. MR 41 \#7066.

4. - Periodic points and invariant measures for Axiom A diffeomorphisms,

Trans. Amer. Math. Soc. 154 (1970), 377-397.

5. — Markov partitions for Axiom A diffeomorphisms, Amer. J. Math. 92 (1970), 725-747. MR 43 \#2740.

6. - Markov partitions and minimal sets for Axiom A diffeomorphisms, Amer. J. Math. 92 (1970), 907-918. MR 43 \#2739.

7. —-, Periodic orbits for hyperbolic flows, Amer. J. Math. 94 (1972), 1-30.

8. W. Feller, An introduction to probability theory and its applications. Vol. I, 3rd ed., Wiley, New York, 1968. MR 37 \#3604.

9. N. A. Friedman and D. S. Ornstein, On isomorphism of weak Bernoulli transformations, Advances in Math. 5 (1970), 365-394.

10. D. S. Ornstein, Factors of Bernoulli shifts are Bernoulli shifts, Advances in Math. 5 (1970), 349-364. MR 43 \#4786.

11. K. R. Parthasarathy, A note on mixing processes, Sankhyā Ser. A 24 (1962), 331-332. MR 29 \#6535.

12. - Probability measures on metric spaces, Probability and Math. Statist., no. 3, Academic Press, New York, 1967. MR 37 \#2271.

13. V. A. Rohlin, Lectures on the entropy theory of transformations with invariant measure, Uspehi Mat. Nauk 22 (1967), no. 5 (137), 3-56=Russian Math. Surveys 22 (1967), no. 5, 1-52. MR 36 \#349.

14. K. Sigmund, Generic properties for invariant measures of Axiom A diffeomorphisms, Invent. Math. 10 (1970), 99-110.

15. - Ergodic averages for Axiom A diffeomorphisms, Z. Wahrscheinlichkeitstheorie und Verw. Gebiete 20 (1971), 319-324.

16. - On the space of invariant measures for hyperbolic flows, Amer. J. Math. (to appear).

17. S. Smale, Differentiable dynamical systems, Bull. Amer. Math. Soc. 73 (1967), 747-817. MR 37 \#3598.

18. R. Williams, Classification of subshifts of finite type (to appear).

Mathematisches Institut der Universität, A-1090 Wien, Strudlhofgasse 4, VienNa, AUstria 